\title{
Outcome of Adjustable Suspensory Fixation for Femoral Graft in ACL Reconstruction
}

Adnan A. Alim Al Sebaie, Mohamed Ibrahim Abulsoud, Ahmed Mohamed Mahmoud Salem

Department of Orthopedic Surgery, Faculty of Medicine, Al-Azhar University, Cairo, Egypt

Corresponding author: Ahmed Mohamed Mahmoud Salem, Mobile: 01027227236;

Email: ahmedsalemortho@gmail.com

\begin{abstract}
Background: The incidence of torn anterior cruciate ligament (ACL) has greatly increased, with today's increasing enthusiasm for sports activities. As a result, reconstruction of the torn anterior cruciate ligament became a common surgical procedure in orthopaedic surgery.

Objective: To evaluate short term clinical outcome of adjustable suspensory fixation for femoral graft in ACL reconstruction.

Methods: All patients treated for ACL reconstruction with an ipsilateral hamstring between March 2017 and March 2018 were evaluated. Subjects were assigned to TightRope ${ }^{\text {TM }}$ (TR) femoral fixation. All patients were evaluated with the Lachman test, pivot-shift test, 2000 International Knee Documentation Committee (IKDC) knee examination. The subjective evaluation was performed using the Lysholm knee score. CT examination was performed to evaluate femoral and tibial tunnels enlargement at four different levels. All patients were assessed at a 12 month follow-up visit. Power analysis was performed a priori in accordance with the femoral and tibial tunnels enlargement values from the CT scans.

Results: The group was homogenous at baseline with regard to age, gender, BMI, dominance and disease duration. At the final follow-up, no statistically significant differences were found according to subjective and objective clinical outcome measures. According to the femoral tunnel enlargement, no statistically significant difference was found between tunnel at operation and 12 months later.

Conclusion: In transtibial ACL reconstruction, the use of adjustable-loop length device products, on the femoral side, led to better clinical and radiological results.
\end{abstract}

Keywords: Tunnel widening, ACL, Femoral tunnel, Adjustable Suspensory fixation device.

\section{INTRODUCTION}

Fruitful foremost cruciate tendon (ACL) remaking relies upon a few variables, including stable beginning obsession, natural bone join coordination, and sufficient unite quality. The join obsession decision is basic for a decent careful outcome. It limits lengthening and counteracts disappointment at the unite connection locales before reconciliation is finished ${ }^{(1)}$.

In any case, the strategy that creates the best outcome stays obscure. As of late, the EndoButton ${ }^{\circledR}(\mathrm{EB}$; Smith and Nephew) has turned out to be one of the most well-known obsession gadgets. It is a suspensory obsession framework, and a few examinations have officially exhibited its mechanical quality ${ }^{(2,3)}$.

The writing reports that one of the hindrances of the suspension framework is the dynamic growth of the femoral bone passage breadth ${ }^{(4)}$. In fact, it was reported that suspensory fixation results in an increased rate of tunnel widening compared with aperture fixation ${ }^{(5)}$.

Recently, a new fixation device, the Tight Rope ${ }^{\mathrm{TM}}$ (TR; Arthrex, Naples, FL, USA), was introduced; it is a second-generation adjustable loop length suspensory fixation device that can be tightened intraoperatively. The adjustable graft loop has a four-point, knotless locking mechanism that relies on multiple points of friction to create resistance to cyclic displacement and slippage under tension. Some authors also believe that the tensioning sutures at the button end reduce the loop length and tension on the graft strands in the same direction of graft advancement into the socket. This allows an optimal graftto-socket fill, reducing longitudinal ('bungee-effect') graft motion within the bone tunnel and optimizing graft-tobone healing ${ }^{(6)}$. In contrast, other authors demonstrated

that the TR shows more slippage and less stiffness compared to the EB. Those mechanical proprieties can most likely facilitate tunnel widening ${ }^{(7,8)}$.

The point of this investigation was to assess clinical outcome and femoral and tibial passages augmentation in patients who experienced ACL transtibial recreation utilizing a customizable circle length suspensory obsession gadget, the TR. The speculation is that the flexible circle gives better outcome. Our essential target was to assess whether an ACL transtibial procedure remaking with the TR produces a femoral passage development utilizing a PC tomography (CT) examine after a subsequent time of a year. The optional goal was to assess whether passage augmenting influences clinical results.

\section{METHODS}

Participants: All patients treated by the two senior authors for ACL reconstruction with ipsilateral semitendinosus (ST) and gracilis (G), between March 2017 March 2018 were retrospectively enrolled in this study. The exclusion criteria for all patients were advanced joint arthritis with complete loss of joint space, age more than 45 years, young patients with open physis and multiple ligaments injury of the knee.

\section{Ethical approval:}

All patients agreed to participate in the study and signed an informed consent form in accordance with the Declaration of Helsinki. The study protocol was 
approved by the Local Ethics and Experimental Research Committee.

Surgical technique: Surgery was performed using an arthroscopic technique through a trans-patellar and anteromedial portal. Surgery was performed either with spinal or general anesthesia according to patient preference. We always prescribed prophylactic antibiotics and anti-thromboembolic medication both pre- and postoperatively. The patient was placed in the supine position. In all patients, we placed an ischemic band at the root of the lower limb. Complete diagnostic arthroscopy was performed on every patient to confirm the ACL tear and to address any meniscal or chondral injuries. Gracilis and semitendinosus tendons were harvested through a small incision made over the pes anserinus and were prepared with bunnell sutures. Both tendons were pretensioned.

The diameters of the grafts were measured in 0.5 $\mathrm{mm}$ steps, and the femoral tunnels were drilled according to the diameters of the grafts that were considered. The extra-articular landmark of the tibial tunnel was always one centimeter above the insertion of the pes anserinus and $1.5 \mathrm{~cm}$ medial to the tibial tubercle. The tibial tunnel was drilled using a standard tibial guide. An impingement rod was used to prevent the femoral roof from impinging on the graft. In the coronal plane, the tibial drill guide was inclined to place one guide-wire at $60^{\circ}$ relative to the medial joint line of the tibia. In the sagittal plane, drill guide inclination was $50^{\circ}$. The intra-articular point of the tibial guide was placed at the center of the native tibial footprint of the ACL.

After insertion of the guide pin, a tibial tunnel was created using a reamer of the same diameter as the graft. After the tibial tunnel was established, an offset guide was placed through the tibial tunnel. The femoral tunnel was drilled through the tibial tunnel with the knee flexed at $90^{\circ}$ on a pin guide located in the center of the anatomical ACL insertion (at 10 o'clock for right knee and two o'clock for left knee), seven millimeter anterior to the posterior margin of the lateral femoral condyle. The tunnel was drilled over the guide pin using a $3.5 \mathrm{~mm}$ drill. The length of the tunnel was then measured, and a femoral tunnel of the necessary length was drilled to place the graft.
The graft was pulled up to the ceiling of the femoral tunnel. The remaining process of tibial fixation was completed without any modification; tibial fixation was completed with a biodegradable screw on the tibial side with a diameter that was one millimeter greater than the tibial tunnel and $30-35 \mathrm{~mm}$ in total length.

Rehabilitation protocol: Patients were allowed to walk using crutches starting on the first day after surgery. A rehabilitation brace was used for all patients for four weeks. Terminal extension and active quadriceps isometric exercises were started immediately postoperatively. Full weight bearing was allowed as soon as it was tolerated. Full flexion and gradual ambulation without the use of the brace were allowed after the fourth postoperative week. Unrestricted return to sports or activity was allowed between five and seven months after surgery.

Clinical evaluation: An objective assessment of stability was performed through the execution of the Lachman test, pivot-shift test and 2000 International Knee Documentation Committee (IKDC) knee examination. Subjective evaluation was performed using the Lysholm knee score. All patients were assessed at a 12 month follow-up visit by an operator who was different from the surgeon.

Radiological evaluation: All patients underwent CT examination with Philips Computerized Tomography (MX 800016 layers; GE Light Speed 16layers) to study and evaluate the change in diameter of the femoral tunnel according to a CT protocol proposed by Iorio $\boldsymbol{e t}$ al. and Vadalà $\boldsymbol{e t}$ al. ${ }^{(9,10)}$. Specifically, image acquisitions were obtained through a volumetric mode; a volume was scanned, and the raw data sets were subsequently manipulated, thus allowing post-process reformation along all of the axes (perpendicular, horizontal, and oblique). All of the diameters were calculated in millimeters. All of the measurements were performed by an expert radiologist who was blinded. Four scans determined the tunnel diameter (Figure 1). The values were compared considering the tunnel measurement (diameter of the drill used for the halftunnel in all patients) at time 0 (T0).
F1

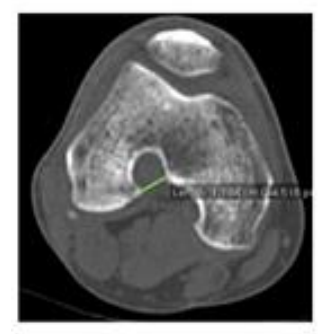

F2

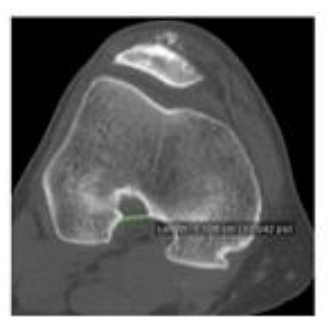

F3

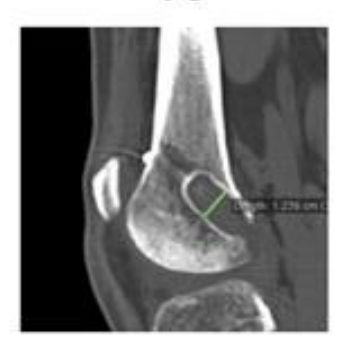

F4

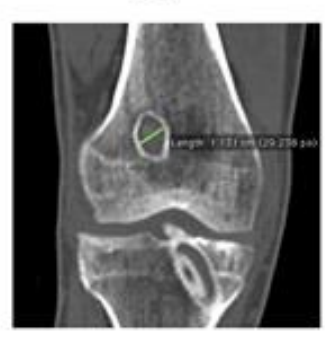

Figure (1): Computer tomography (CT) scan evaluation at four different levels: F1: femoral tunnel at the notch, axial image; F2: femoral tunnel at middle third, axial image; F3: femoral tunnel in the middle point, on sagittal image reconstruction; F4: femoral tunnel in the middle point, on coronal image reconstruction.

Sample size: Power analysis was performed a priori in accordance with the femoral tunnel enlargement values from the CT scans. Assuming a two-tailed $\alpha$ value of 0.05 (sensitivity $95 \%$ ), a $\beta$ value of 0.20 (study power: $80 \%$ ), and an effect size 
value of 0.80 , we determined that at least 25 patients were required.

\section{Statistical analysis}

Recorded data were analyzed using the statistical package for social sciences, version 20.0 (SPSS Inc., Chicago, Illinois, USA). Quantitative data were expressed as mean \pm standard deviation (SD). Qualitative data were expressed as frequency and percentage.

\section{The following tests were done:}

- Independent-samples t-test of significance was used when comparing between two means.

- P-value <0.05 was considered significant.

\section{RESULTS}

The overall results: The demographic data of the patients regarding age and sex are illustrated in tables $(1,2)$.

Table (1): Description of age in studied patients.

\begin{tabular}{|c|c|c|}
\hline \multicolumn{2}{|c|}{ Variables } & $\begin{array}{c}\text { Studied patients } \\
(\mathrm{N}=\mathbf{2 5})\end{array}$ \\
\hline \multirow{5}{*}{ Age(years) } & Mean & 27.36 \\
\hline & \pm SD & 5.04 \\
\hline & Min & 21 \\
\hline & Max & 36 \\
\hline & Range & $(21-36)$ \\
\hline
\end{tabular}

Table (2): Description of sex in studied patients.

\begin{tabular}{|l|l|c|c|}
\hline \multicolumn{2}{|l|}{ Variables } & \multicolumn{2}{c|}{$\begin{array}{c}\text { Studied patients } \\
(\mathbf{N}=25)\end{array}$} \\
\hline \multirow{2}{*}{ Sex $(\mathbf{n}, \%)$} & Male & 23 & $92 \%$ \\
\cline { 2 - 4 } & Female & 2 & $8 \%$ \\
\hline
\end{tabular}

Lysholm score: patient were evaluated using Lysholm score table (3) mean 93.8 which is excellent outcome and IKDC score table (4)
Table (3): Description of Lysholm knee score in studied patients.

\begin{tabular}{|l|l|c|c|}
\hline \multicolumn{2}{|c|}{ Variables } & \multicolumn{2}{c|}{$\begin{array}{c}\text { Studied } \\
\text { patients } \\
\text { (N= 25) }\end{array}$} \\
\hline \multirow{2}{*}{ Limp } & $\mathbf{3}$ & 1 & $4 \%$ \\
\cline { 2 - 4 } & No & 24 & $96 \%$ \\
\hline Crutch & No & 25 & $100 \%$ \\
\hline Locking & No & 25 & $100 \%$ \\
\hline \multirow{3}{*}{ G. way } & $\mathbf{1 5}$ & 1 & $4 \%$ \\
\cline { 2 - 4 } & $\mathbf{2 0}$ & 1 & $4 \%$ \\
\cline { 2 - 4 } & No & 23 & $92 \%$ \\
\hline \multirow{3}{*}{ Pain } & $\mathbf{1 5}$ & 4 & $16 \%$ \\
\cline { 2 - 4 } & $\mathbf{2 0}$ & 10 & $40 \%$ \\
\cline { 2 - 4 } & No & 11 & $44 \%$ \\
\hline \multirow{3}{*}{ Swelling } & $\mathbf{6}$ & 9 & $36 \%$ \\
\cline { 2 - 4 } & No & 16 & $64 \%$ \\
\hline Climb Stair & No & 25 & $100 \%$ \\
\hline \multirow{3}{*}{ Squat } & $\mathbf{1}$ & 2 & $8 \%$ \\
\cline { 2 - 4 } & $\mathbf{4}$ & 4 & $16 \%$ \\
\cline { 2 - 4 } & No & 19 & $76 \%$ \\
\hline \multirow{3}{*}{$\%$} & Mean & \multicolumn{2}{|c}{$93.8 \pm 5.6$} \\
\hline & \pm SD & \multicolumn{2}{|c}{80} \\
\cline { 2 - 4 } & Min & Max & \multicolumn{2}{|c}{100} \\
\cline { 2 - 4 } & & \multicolumn{2}{|c|}{} \\
\hline
\end{tabular}

Table (4): Description of IKDC Score in studied patients.

\begin{tabular}{|l|c|c|c|}
\hline \multicolumn{2}{|c|}{ Variables } & \multicolumn{2}{c|}{$\begin{array}{c}\text { Studied patients } \\
(\mathbf{N}=\mathbf{2 5})\end{array}$} \\
\hline $\begin{array}{l}\text { IKDC } \\
\text { Score } \\
(\mathbf{N}, \boldsymbol{\%})\end{array}$ & A & 20 & $80 \%$ \\
\cline { 2 - 4 } & B & 5 & $20 \%$ \\
\hline
\end{tabular}

This table shows the description of IKDC Score in studied patients.

CT scan is done to the patient to assess femoral and tibial tunnel widening as shown in table (5) and (6) respectively.

Table (5): Comparison between femoral tunnel follows up in studied patients.

\begin{tabular}{|c|c|c|c|c|c|c|}
\hline \multirow{2}{*}{\multicolumn{2}{|c|}{ Variables }} & \multirow{2}{*}{ At op. } & \multicolumn{3}{|c|}{ CT } & \multirow{2}{*}{ P-value } \\
\hline & & & Axial & Sagittal & Coronal & \\
\hline Femoral & Mean & 8.64 & 8.88 & 8.89 & 8.96 & $\begin{array}{l}\mathrm{P} 1=0.47 \\
\mathrm{P} 2=0.26\end{array}$ \\
\hline
\end{tabular}




\begin{tabular}{|l|l|l|l|l|l|l|}
\hline & \pm SD & 0.49 & 0.87 & 0.76 & 0.85 & $\begin{array}{l}\text { P3 }=0.25 \\
\text { P4 }\end{array}$ \\
\hline
\end{tabular}

This table shows: No statistical significant difference between femoral tunnel follows up in studied patients (P1). No statistical significant difference between femoral tunnel follows at operation and axial, sagittal, and coronal $\mathrm{CT}$ (P2, 3, and 4 respectively) follow up in studied patients.

Table (6): Comparison between tibial follows up in studied patients.

\begin{tabular}{|c|c|c|c|c|c|c|}
\hline \multirow{2}{*}{\multicolumn{2}{|c|}{ Variables }} & \multirow{2}{*}{ At op. } & \multicolumn{3}{|c|}{ CT } & \multirow{2}{*}{ P-value } \\
\hline & & & Axial & Sagittal & Coronal & \\
\hline \multirow{2}{*}{ Tibial } & Mean & 9.84 & 10.38 & 10.10 & 10.15 & \multirow{2}{*}{$\begin{array}{l}\mathrm{P} 1=0.66 \\
\mathrm{P} 2=0.21 \\
\mathrm{P} 3=0.55 \\
\mathrm{P} 4=0.47\end{array}$} \\
\hline & $\pm \mathrm{SD}$ & 0.37 & 0.91 & 2.02 & 2.09 & \\
\hline
\end{tabular}

This table shows: No statistical significant difference between tibial tunnel follows up in studied patients (P1). No statistical significant difference between tibial tunnel follows at operation and axial, sagittal, and coronal $\mathrm{CT}$ (P2, 3, and 4 respectively) follow up in studied patients.

\section{DISCUSSION}

The most important finding of this study was that there was no difference in femoral and tibial tunnels widening between operation time and one year later. No difference was observed regarding the other clinical patient-reported and clinical examination outcomes.

To the best of our knowledge, this is the first report that analyzed tunnel widening with the TR system. Tunnel enlargement after ACL reconstruction is not yet fully understood, although it is the main focus of many studies. Many authors showed that tunnel enlargement is more evident in the femoral tunnel than in the tibial tunnel ${ }^{(11-13)}$.

It is definitely determined by biological and mechanical factors, such as the micromovements of the graft in the tunnel, including the longitudinal (bungee-effect) and transverse (windshield-wipereffect) graft motions within the bone tunnel, which in turn may lead to bone tunnel dilation ${ }^{(\mathbf{1 4})}$.

According to many authors, the causes are the rigidity of the fixation device ${ }^{(\mathbf{9 , 1 5})}$ or suspension devices causing graft motion in the femoral tunnel $(11,14,16,17)$.

There are different radiological protocols for the study of femoral tunnel enlargement, including radiographs (18-20) and magnetic resonance (21); however, we chose a protocol with a CT exam because it appears to be the most accurate method, as demonstrated by Marchant et al. (22) and Rathnayaka ${ }^{(23)}$.

The decision to choose a follow-up period of 12 months was because this was the period in which most femoral tunnel widening occurred. Peyrache $\boldsymbol{e t}$ al. ${ }^{(24)}$ reported a tunnel dilation of $16 \%$ three months after surgery with no diameter change for up to two years and a reduction of seven percent over three years. Webster et al. (20) showed that the enlargement of the femoral tunnel is more evident in the four months following surgery without any change in diameter for up to two years after the surgery.

Finally, in a recent work in MR, Weber ${ }^{(25)}$ showed that there is a progressive increase of femoral tunnel diameter from the sixth to the $24^{\text {th }}$ week after surgery, continuing to increase up to 12 months after surgery and reducing slightly after 24 months postoperatively. We performed a single measurement after 12 months that should be indicative of the maximum postoperative enlargement.

\section{CONCLUSION}

This study suggests that there is no significant difference of tunnels widening of adjustable suspensory fixation for femoral graft in ACL reconstruction and excellent stability and functional and clinical outcome provided using careful patients selection, proper surgical technique and adequate rehabilitation program.

\section{REFERENCES}

1. Brand J, Weiler A, Caborn DN et al. (2000): Graft fixation in cruciate ligament reconstruction. Am J Sports Med., 28(5):761-74.

2. Ahmad CS, Gardner TR, Groh M et al. (2004): Mechanical properties of soft tissue femoral fixation devices for anterior cruciate ligament reconstruction. Am J Sports Med., 32(3):635-40.

3. Uchio $Y$, Ochi M, Sumen $Y$ et al. (2002): Mechanical properties of newly developed loop ligament for connection between the EndoButton and hamstring tendons: comparison with Ethibond sutures and endobutton tape. J Biomed Mater Res., 63(2):173-81.

4. Jansson KA, Harilainen A, Sandelin $\mathrm{J}$ et al. (1999): Bone tunnel enlargement after anterior cruciate ligament reconstruction with the hamstring 
autograft and endobutton fixation technique. A clinical, radiographic and magnetic resonance imaging study with 2 years follow-up. Knee Surg Sports Traumatol Arthrosc., 7(5):290-5.

5. Barber FA, Spruill B, Sheluga $M$ (2003): The effect of outlet fixation on tunnel widening. Arthroscopy, 19:485-92.

6. Lubowitz JH, Ahmad CS, Anderson K (2011): All-inside anterior cruciate ligament graft-link technique: second-generation, no-incision anterior cruciate ligament reconstruction. Arthroscopy, 27(5):717-27.

7. Johnson JS, Smith SD, LaPrade CM et al. (2015): A biomechanical comparison of femoral cortical suspension devices for soft tissue anterior cruciate ligament reconstruction under high loads. Am J Sports Med., 43(1):154-60.

8. Petre BM, Smith SD, Jansson KS et al. (2013): Femoral cortical suspension devices for soft tissue anterior cruciate ligament reconstruction: a comparative biomechanical study. Am J Sports Med., 41(2):416-22.

9. Iorio R, Di Sanzo V, Vadalà A et al. (2013): ACL reconstruction with hamstrings: how different technique and fixation devices influence bone tunnel enlargement. Eur Rev Med Pharmacol Sci., 17(21):2956-61.

10. Vadalà A, Iorio R, De Carli A et al. (2013): Platelet-rich plasma: does it help reduce tunnel widening after ACL reconstruction? Knee Surg Sports Traumatol Arthrosc., 21(4):824-9.

11. Clatworthy MG, Annear $\mathrm{P}$, Bulow JU et al. (1999): Tunnel widening in anterior cruciate ligament reconstruction: a prospective evaluation of hamstring and patella tendon grafts. Knee Surg Sports Traumatol Arthrosc., 7(3):138-45.

12. Ma CB, Francis $K$, Towers $J$ et al. (2004): Hamstring anterior cruciate ligament reconstruction: a comparison of bioabsorbable interference screw and endobutton-post fixation. Arthroscopy, 20(2):122-8

13. Nebelung W, Becker R, Merkel M et al. (1998): Bone tunnel enlargement after anterior cruciate ligament reconstruction with semitendinosus tendon using endobutton fixation on the femoral side. Arthroscopy, 14(8):810-5.

14. Höher J, Livesay GA, Ma CB et al. (1999): Hamstring graft motion in the femoral bone tunnel when using titanium button/polyester tape fixation. Knee Surg Sports Traumatol Arthrosc., 7(4):215-9.

15. Tsuda E, Fukuda Y, Loh JC et al. (2002): The effect of soft-tissue graft fixation in anterior cruciate ligament reconstruction on graft-tunnel motion under anterior tibial loading. Arthroscopy, 18(9):960-7.

16. L'Insalata JC, Klatt B, Fu FH et al. (1997): Tunnel expansion following anterior cruciate ligament reconstruction: a comparison of hamstring and patellar tendon autografts. Knee Surg Sports Traumatol Arthrosc., 5(4):234-8.

17. Muneta T, Sekiya I, Yagishita K et al. (1999): Two-bundle reconstruction of the anterior cruciate ligament using semitendinosus tendon with endobuttons: operative technique and preliminary results. Arthroscopy, 15(6):618-24.

18. Choi NH, Lee JH, Son KM et al. (2010): Tibial tunnel widening after anterior cruciate ligament reconstructions with hamstring tendons using Rigidfix femoral fixation and Intrafix tibial fixation. Knee Surg Sports Traumatol Arthrosc., 18(1): 92-7.

19. Segawa H, Omori G, Tomita S et al. (2001): Bone tunnel enlargement after anterior cruciate ligament reconstruction using hamstring tendons. Knee Surg Sports Traumatol Arthrosc., 9(4):206-10.

20. Webster KE, Feller JA, Hameister KA (2001): Bone tunnel enlargement following anterior cruciate ligament reconstruction: a randomised comparison of hamstring and patellar tendon grafts with 2-year follow-up. Knee Surg Sports Traumatol Arthrosc., 9(2):86-91.

21. Järvelä T, Moisala AS, Paakkala T et al. (2008): Tunnel enlargement after doublebundle anterior cruciate ligament reconstruction: a prospective, randomized study. Arthroscopy, 24(12):1349-57.

22. Marchant MH, Willimon SC, Vinson E et al. (2010): Comparison of plain radiography, computed tomography, and magnetic resonance imaging in the evaluation of bone tunnel widening after anterior cruciate ligament reconstruction. Knee Surg Sports Traumatol Arthrosc., 18(8):1059-64.

23. Rathnayaka K, Momot KI, Noser H et al. (2012): Quantification of the accuracy of MRI generated 3D models of long bones compared to CT generated 3D models. Med Eng Phys., 34(3):357-63.

24. Peyrache MD, Djian P, Christel $P$ et al. (1996): Tibial tunnel enlargement after anterior cruciate ligament reconstruction by autogenous bone-patellar tendon-bone graft. Knee Surg Sports Traumatol Arthrosc., 4(1):2-8.

25. Weber AE, Delos D, Oltean HN et al. (2016): Tibial and femoral tunnel changes after ACL reconstruction: a prospective 2-year longitudinal MRI study. Am J Sports Med., 43(5):1147-56. 